\title{
Per sviluppare la pratica clinica è necessario più lavoro familiare e più lavoro all'interno del sistema. Commento a Parrella, Riefolo e Vincenti
}

\author{
Jaakko Seikkula*
}

Mi è piaciuto molto leggere i commenti profondi e analitici di Carmine Parrella, Giuseppe Riefolo ed Enrico Vincenti. Nel mio commento sulle loro riflessioni voglio concentrarmi su alcune questioni specifiche. Sono felice che abbiano esposto commenti importanti sulla parte umanistica della pratica che non è necessario ripetere.

Mi fa piacere anche che tutti e tre abbiano segnalato aspetti diversi del mio articolo sulla pratica psicoterapeutica e psichiatrica in generale. Per esempio, Carmine Parrella ha paragonato l'Open Dialogue (OD) con la riforma di Basaglia in Italia. Questa è una questione su cui ho pensato molto ultimamente ed è stata sollevata in alcuni progetti di OD in Italia. Mentre la riforma psichiatrica in Italia era rivolta principalmente ai diritti umani dei pazienti psichiatrici, l'Open Dialogue è fortemente basato sullo sviluppare una psicoterapia per le più gravi malattie mentali. Nei progetti che stiamo portando avanti abbiamo visto un'integrazione di queste due prospettive importanti, e credo che entrambe le parti abbiano ottime ragioni.

Parrella ha fatto riferimento all'importanza della formazione nel pensiero di Basaglia. La formazione sembra veramente aiutarci a trovare un punto di incontro più umano con le persone che hanno disturbi psicotici. Tuttavia, vedo che manca ancora qualcosa. E - credo - a questo l'OD può contribuire con le sue radici psicoterapeutiche. A volte non basta rispettare i diritti umani ed essere democratici, bisogna formarsi per tollerare le strane conversazioni che si possono avere con coloro che definiamo pazienti. La sfida può includere la necessità di gestire la violenza o la minaccia di violenza del paziente verso altri membri della famiglia. Oppure trovarsi in qualsiasi tipo di situazione estrema.

Vincenti ha analizzato le diverse parti della pratica di OD, commentando

\footnotetext{
*Jaakko Seikkula, Dipartimento di Psicologia, Università di Jyväskylä, Finlandia. E-mail: jaakko.seikkula@jyu.fi
} 
inoltre quali siano secondo lui gli elementi che mancano nell'OD. Scrive: 'Penso comunque sia necessario fare un passo ulteriore per valorizzare al massimo il contributo dell'autore. Prendere in considerazione il contesto permette di inserire la persona sofferente all'interno del suo ambiente di vita e quindi, dal mio punto di vista, permette di cogliere nelle sue relazioni la funzionalità che esse hanno nel mantenere le soluzioni storiche che l'hanno configurato.'

Dopo questa affermazione Vincenti, in modo gentile, inizia ad introdurre il suo punto di vista dicendo che non è solo dialogo, ma che servirebbe un'analisi e una teoria su quello che chiamiamo paziente e sulle relazioni che sta vivendo. Egli propone anche alcuni esempi di casi clinici. Voglio parlare di questa affermazione perché penso che sia proprio al cuore della pratica dialogica e la lezione che ho ricevuto nel fare ricerca e nell'essere coinvolto nella pratica clinica all'interno del quadro dialogico. Secondo le mie esperienze, sorprendentemente, meno 'analizziamo' e meglio è. Penso che i risultati a lungo termine dei pazienti che vent'anni prima hanno avuto esperienza con l'OD confermino questo (Bergström et al., 2018).

Sono d'accordo che abbiamo bisogno di alcuni concetti e teorie per analizzare la nostra pratica per capire e imparare. Ma allo stesso tempo non credo più che dobbiamo analizzare il sistema dei nostri clienti nella pratica clinica. L'elemento eccezionale della pratica dialogica è il fatto che non diamo mai nulla per scontato ma - invece - ogni situazione è in un flusso continuo di cambiamento. Nei dialoghi dei nostri pazienti, anche nei primi minuti, i genitori possono imparare nuovi modi di relazionarsi con i loro figli. Ricordo in particolare l'incontro con una madre e un padre ed il loro figlio di 32 anni che era psicotico da 16 anni; era il nostro primo incontro. I genitori stavano facendo molta fatica con il figlio, il suo abuso di droghe, i suoi comportamenti estremi e così via. Ma erano così sorpresi quando con i miei co-terapeuti alla fine della sessione, nella nostra conversazione riflessiva, abbiamo sottolineato che loro sembravano essere stati così affettuosi dopo tutti questi anni e che da loro non avevamo sentito una parola negativa sul loro figlio. Loro hanno potuto imparare qualcosa di nuovo su sé stessi dai nostri dialoghi e suppongo che questa novità abbia reso possibile la mobilizzazione di altre loro risorse per affrontare la situazione. Quando pensavamo alla famiglia come terapeuti sistemico-familiari (che è la formazione che ricevetti nei primi anni ' 80 ) so che sarebbe stato molto facile trovare delle regole sistemiche non-funzionanti all'interno di questa famiglia, ma non penso che questo li avrebbe aiutati in alcun modo. Al contrario, avrebbe diretto la nostra attenzione agli elementi non-funzionanti della famiglia e non sarebbe stata mobilizzata alcuna nuova risorsa.

Lavorare con le famiglie si è mostrato essere veramente impegnativo da un punto di vista psicodinamico. Forse un motivo di ciò è che esiste una teoria sul mondo interno dei pazienti psicotici. I problemi interni riguardano le esperienze di relazione dei pazienti e certamente la parte principale delle esperienze interne 
più importanti si sviluppano all'interno di una famiglia. In questo esempio, il paziente spesso vuole proteggersi da relazioni troppo strette. A questo punto emergono delle difficoltà nel vedere l'importanza del lavoro con la famiglia. Molti psicoterapeuti individuali prendono la decisione di lavorare solo con il paziente, che dà risultati molto limitati in almeno due modi. Prima di tutto, questa attitudine non aiuta a trovare soluzioni ai problemi della famiglia. In secondo luogo, le risorse psicologiche che vivono all'interno delle relazioni tra i membri della famiglia non saranno mobilizzate. L'OD ha introdotto un modo per affrontare le situazioni famigliari problematiche anche tramite l'accettazione delle voci contraddittorie dei membri della famiglia, poiché la questione principale in OD è sempre generare dialogo, e non trovare soluzioni.

Anche Riefolo fa un commento critico sull' OD quando dice 'Un limite che riconosco è di almeno due ordini. Un primo limite è che mi sembra che l'OD si fermi alla 'condivisione e solidarietà' della sofferenza psicotica, senza coglierne la dimensione della comunicazione transferale che - secondo un registro psicotico - chiede al terapeuta di conoscere intimamente, potrei dire somaticamente (nel senso di un registro pre-simbolico, che magari si evidenzia attraverso l'azione, l'impotenza, il fallimento, gli errori e, quindi, gli enactment) quale sia la natura specifica della sofferenza psicologica del paziente in quel preciso momento e con quel soggetto. Un altro limite che sempre da psicoanalista - trovo nell'approccio dell'OD è una sorta di idealizzazione della 'buona pratica' rappresentata nella dimensione soprattutto concreta.' (Riefolo p. 2)

Devo dire che non ho compreso questo commento fino in fondo. Se ho capito bene, Riefolo dice che nell'OD le esperienze psicotiche non sono condivise solidalmente. Se questo è corretto allora devo scusarmi perché forse il mio articolo non era chiaro su questo punto. La condivisione in modo incarnato della sofferenza dei nostri clienti - incluso quello che chiamiamo paziente e i membri della sua famiglia - è proprio al cuore della pratica dialogica. Mi piace molto che Riefolo parli delle parti 'somatiche' dell'esperienza del terapeuta. Ho usato la parola 'incarnazione' quando mi riferisco al fatto che una piccola parte del processo terapeutico accade tramite parole e in formulazioni esplicite delle esperienze e problemi. Nel dialogo non è nostro scopo fare interpretazioni o dare altri tipi di significati a ciò che dicono i nostri clienti, ma invece di condividere la loro esperienza e insieme creare un nuovo linguaggio della loro esperienza che vive nell'esperienza incarnata che non è ancora stata espressa. Per me è decisamente importante rimanere nel discorso dei nostri clienti, fino al punto che cerco di evitare le parafrasi dei loro commenti, piuttosto ripeto parola per parola ciò che dicono e chiedo loro di approfondire questi concetti. La parte 'curativa' del dialogo risiede proprio in questo: quando includono nuove parole oltre a ciò che gli ha ripetuto il terapeuta sulla loro esperienza, le persone ascoltano e imparano di più su sé stessi. 
La critica all'OD come idealizzazione della 'buona pratica' è strana per me. Se volessi essere critico sulla pratica psicoterapeutica direi questo: gli psicoanalisti e gli psicoterapeuti spesso stanno al di fuori del pensiero pratico psichiatrico e il loro contributo migliore è fare psicoterapia solamente e principalmente nello studio privato, e solo a volte nei servizi pubblici.

In questo modo, il controllo della pratica è dato alle persone che in realtà non capiscono l'importanza dell'attitudine psicoterapeutica nelle cure. Affermo che una parte del successo dell'OD sta esattamente nel trovare modi di integrare l'organizzazione di noi stessi nella pratica clinica e la pratica clinica negli incontri dialogici. Questa non è un'idealizzazione della 'buona pratica' perché nelle ricerche ci siamo concentrati molto sulle difficoltà, sui fallimenti e su altri tipi di sfide. La pratica si sviluppa solo tramite l'analisi della pratica stessa da parte dei professionisti stessi.

Riefolo fa inoltre un'analisi interessante dell'importanza dei fattori 'specifici' e 'aspecifici' della pratica clinica. Probabilmente dobbiamo dire che nell'OD il fattore specifico è l'aspecificità. Il dialogo stesso è lo scopo della pratica. Questo è differente se lo paragoniamo ad altri metodi psicoterapeutici: il dialogo è visto come strumento per realizzare qualcosa. Per esempio, un terapeuta potrebbe pensare che nel buon dialogo emergano più opzioni per parlare delle questioni più difficili nella vita dei pazienti e che all'interno di un buon dialogo si possono fare delle interpretazioni più profonde per aumentare la comprensione di sé stessi. Nella pratica dialogica penso che le nuove esperienze emergano nelle nuove relazioni dialogiche e non è necessario altro in aggiunta.

\section{BIBLIOGRAFIA}

Bergström, T., Seikkula, J., Alakare, B., Mäki, P., Köngäs-Saviaro, P., Taskila, J., Tolvanen, A., \& Aaltonen, J. (2018). The family-oriented Open Dialogue approach in the treatment of first-episode psychosis: Nineteen-year outcomes. Psychiatry Research, 270, 168-17.

Conflitto di interessi: l'autore dichiara che non vi sono potenziali conflitti di interessi.

Approvazione etica e consenso a partecipare: non necessario.

Ricevuto per la pubblicazione: 8 novembre 2021 .

Accettato per la pubblicazione: 8 novembre 2021 .

${ }^{C}$ Copyright: the Author(s), 2021

Licensee PAGEPress, Italy

Ricerca Psicoanalitica 2021; XXXII:602

doi:10.4081/rp.2021.602

This article is distributed under the terms of the Creative Commons Attribution

Noncommercial License (by-nc 4.0) which permits any noncommercial use, distribution,

and reproduction in any medium, provided the original author(s) and source are credited. 